\title{
Expression of the nuclear receptor tailless is required for generation and maintenance of adult neural stem cells and participates in brain tumor generation
}

\author{
Hai-Kun Liu ${ }^{1}$, Günther Schütz ${ }^{1}$ \\ ${ }^{I}$ Molecular Biology of the Cell I, German Cancer Research Center, Im Neuenheimer Feld 280, 69120 Heidelberg, Germany
}

The tailless (Tlx) gene encodes an orphan nuclear receptor which is expressed in the developing eye and brain and in adult neural stem cells. We show that in the adult brain it is exclusively expressed in astrocyte-like B cells of the subventricular zone whereas $C$ and $A$ cells do not express Tlx. These cells have the ability for selfrenewal and are multipotent. Use of a genetic approach allowed the establishment of a cell lineage relationship of neural stem cells from neural epithelial to radial glial cells and finally to adult neural stem cells. Tlx expressing cells express CD133, a marker of brain tumor stem cells, and loss of Tlx leads to upregulation of PTEN in the subventricular zone compatible with its function in control of neural stem cell proliferation. These findings establish Tlx as an indicator for and regulator of adult neural stem cells.

Cell Research (2008) 18:s43. doi: 10.1038/cr.2008.133; published online 4 August 2008 